\begin{tabular}{|c|c|}
\hline Title & Cathodic Reduction of A nodic Oxide Films Formed on Titanium \\
\hline Author(s) & Ohtsuka, Toshiaki; Masuda, Minoru; Sato, Norio \\
\hline Citation & $\begin{array}{l}\text { J. Electrochemical Society, 134(10), 2406-2410 } \\
\text { https://doi.org/10.1149/1.2100212 }\end{array}$ \\
\hline Issue Date & $1987-10$ \\
\hline Doc URL & http:/hdl.handle.net/2115/62191 \\
\hline Rights & $\begin{array}{l}\text { () The Electrochemical Society, Inc. 1987. All rights reserved. Except as provided under U.S. copyright law, this work } \\
\text { may not be reproduced, resold, distributed, or modified without the express permission of The Electrochemical Society } \\
\text { (ECS). The archival version of this work was published in J. Electrochem. Soc. } 1987 \text { volume 134, issue 10, } 2406-2410 \text {. }\end{array}$ \\
\hline Type & article \\
\hline File Information & J.Electrochem.Soc.134,2406-2410(1987), Cathodic reduction --titanium.pdf \\
\hline
\end{tabular}

Instructions for use 


\title{
Cathodic Reduction of Anodic Oxide Films Formed on Titanium
}

\author{
Toshiaki Ohtsuka, Minoru Masuda, and Norio Sato* \\ Electrochemistry Laboratory, Faculty of Engineering, Hokkaido University, Sapporo 060, Japan
}

\section{ABSTRACT}

Cathodic reduction behavior of the anodic oxide film on titanium has been investigated by using ellipsometry combined with electrochemistry. In acidic sulfate solution, the anodic oxide film reductively dissolves into the solution as Ti(III) ion, resulting in the thinning of its thickness without any significant change of the optical property of the remaining film. In neutral phosphate solution, the anodic oxide film absorbs hydrogen in the hydrogen evolution potential region, resulting in a change of the optical property without thinning its thickness. The amount of hydrogen absorbed per unit volume of the film does not depend on the film thickness but on the cathodic potential. The composition change estimated from measurements of anodic charge during the hydrogen release process indicates that the hydrogen absorption begins to occur at about $-0.25 \mathrm{~V}$ (vs. $\mathrm{RHE}$ ) and that the anodic film changes in its composition from $\mathrm{TiO}_{2}$ to $\mathrm{TiOOH}$ at $-0.9 \mathrm{~V}$. The hydrogen absorption induces a decrease of the refractive index and an increase of the extinction index of the anodic film.

Titanium is one of the materials exhibiting the high corrosion resistivity caused by formation of a protective oxide film in oxidative environments (1). However, if titanium is placed under cathodic bias conditions or in reduetive environments, the oxide film changes in its property because of the hydrogen attack.

Under cathodic bias conditions, hydrogen is absorbed into the titanium metal through a modified oxide film layer, resulting in hydrogen brittleness. Fukuzuka et al. (2) have pointed out that the oxide film formed by air oxidation at elevated temperatures acts as a barrier against the hydrogen absorption into the titanium metal but that the oxide film anodically formed loses its barrier property during cathodic reduction in a time period shorter than that of the air oxidation film. Dyer et $a l$. (3) have suggested that hydrogen can be absorbed by the anodic oxide film in the hydrogen evolution potential region and that hydrogen in the oxide film can be reversibly desorbed in the anodic potential region. They observed, using ellipsometry, a significant change of optical property of the films during the electrochemical hydrogen absorption-desorption process. They also observed the electrode impedance response, which changes depending on the cathodic potential (4).

For a photoelectrochemical electrode of $\mathrm{TiO}$, it has been reported that the electrochemical reduction treatment markedly influences the semiconductive property of the $\mathrm{TiO}_{2}$, resulting in an increase of photoresponse current $(5,6)$. The hydrogen absorption process of $\mathrm{TiO}_{2}$ has also been discussed from a viewpoint of electrochromic reactions by Ohzuku et al. (7) and of hydrogen gas detectors by Horrin (8). These results indicate that $\mathrm{TiO}_{2}$ changes its color and electrical conductivity, if hydrogen is absorbed in the $\mathrm{TiO}_{2}$.

In this paper, the optical property and composition of the anodic oxide film on titanium during cathodic reduction has been investigated by ellipsometry and coulometry.

\section{Experimental}

The titanium electrode used was a sheet $99.85 \%$ pure and $0.5 \mathrm{~mm}$ thick with a small tub for electrical connection. The pretreatment of the electrode was the same as that described in a previous paper (1).

The electrolytes were a $0.1 \mathrm{~mol} \mathrm{dm^{-3 }}$ sulfuric acid and a phosphate buffer solution (a mixture of $0.1 \mathrm{~mol} \mathrm{dm}^{-3}$ $\mathrm{Na}_{2} \mathrm{HPO}_{4}$ and $0.1 \mathrm{~mol} \mathrm{dm} \mathrm{d}^{-3} \mathrm{NaH}_{2} \mathrm{PO}_{4}$ ) prepared from analytical grade reagents and redistilled water. Before use in experiments, the electrolytes were deoxygenated by bubbling purified gaseous nitrogen in electrolyte reservoirs connected to the electrochemical cell.

The ellipsometer used was of the horizontal type (Mizojiri Type DV-36). The manual standard null method was adopted for measuring $\Psi$ and $\Delta$ with a light of wavelength $\lambda=546.1 \mathrm{~nm}$ at an angle of incidence $\phi=70.36$.

The experimental temperature was $25.0^{\circ} \pm 0.2^{\circ} \mathrm{C}$. The potential was measured in reference to a $\mathrm{Ag} / \mathrm{AgCl}$ elec-

*Electrochemical Society Active Member. trode and converted into the reversible hydrogen electrode scale in the same solution at $P_{\mathrm{H}_{2}}=1 \mathrm{~atm}$ (RHE). Titanium ions dissolved into the electrolytes during cathodic reduction were analyzed by the colorimetric method using tiron.

\section{Results}

Cathodic reduction in acidic solution.-Figure 1 shows the result of ellipsometric measurements during the open-circuit decay for $100 \mathrm{~min}$ and the following galvanostatic reduction at $-10 \mu \mathrm{A} \mathrm{cm}^{-2}$ of an anodic oxide

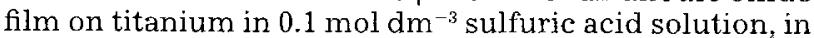
which the loci of the ellipsometric parameters, $\Psi$ and $\Delta$, are plotted. The original anodic oxide film, $18.0 \mathrm{~nm}$ thick, was formed at $E=5.65 \mathrm{~V}$ (vs. RHE) for $1 \mathrm{~h}$ in the phosphate buffer solution at $p \mathrm{H} \mathrm{6.9}$. The loci in Fig. 1 change with time along a theoretical line which is computed with the complex refractive indexes of the oxide film, $n_{\mathrm{t}}$ $=2.10-0.03 \mathrm{i}$, and of the titanium substrate, $n_{\mathrm{s}}=2.7-2.9 \mathrm{i}$ (1), as a function of the film thickness. The result shows that the oxide film is thinned in the acidic sulfuric solution during the open-circuit or the galvanostatic reduction without any significant change of its optical property. The decay of $\Psi$ and $\Delta$ during the open circuit for 100 min indicated that the oxide film was thinned gradually at a rate of about $1 \mathrm{~nm} \mathrm{~h} \mathrm{~h}^{-1}$ in the acidic sulfate solution. The decay rate of $\Psi$ and $\Delta$ was found to be much higher for galvanostatic reduction than for open circuit, which

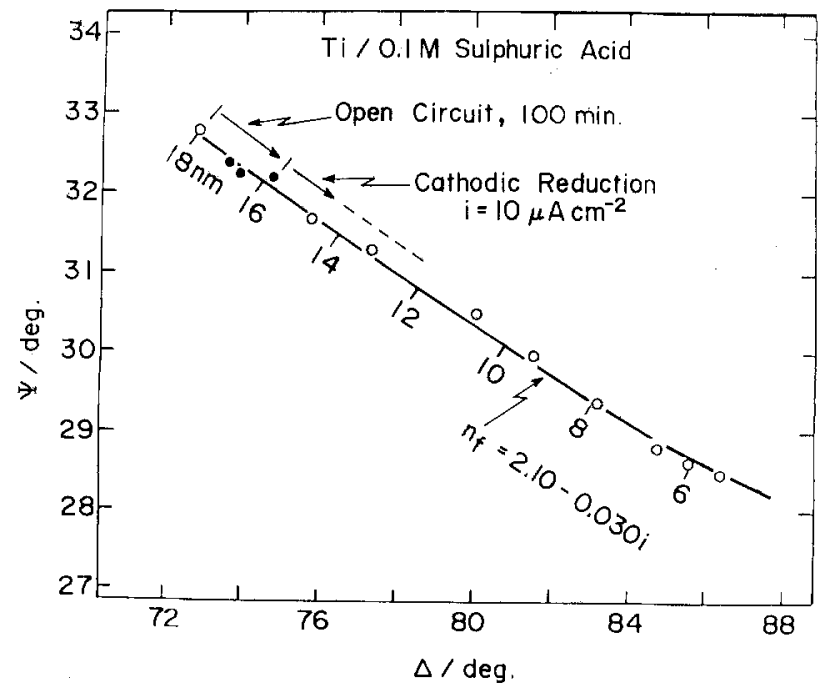

Fig. 1. Change in ellipsometric parameters $\Psi$ and $\Delta$ of the oxide film during cathodic reduction at $i=-10 \mu \mathrm{A} \mathrm{cm}^{-2}$ and subsequent opencircuit decay for $100 \mathrm{~min}$ in $0.1 \mathrm{~mol} \mathrm{dm}{ }^{-3}$ sulfuric acid solution. The curve indicates a theoretical line computed as a function of film thickness for a film with the complex refractive index, $n_{f}=2.10-0.03 i$, on the titanium substrate with $n_{s}=2.7-2.9$. The original oxide film was anodically formed by potentiostatic ih oxidation at $E=5.65 \mathrm{~V}$ (vs. $\mathrm{RHE}$ ) in phosphate buffer solution at $\mathrm{pH} 6.9$. 
indicates that the rapid thinning can take place under cathodic bias conditions.

The decrease of the film thickness accompanies the dissolution of titanium ion. Figure 2 shows the result of cathodic reduction at $-20 \mu \mathrm{A} \mathrm{cm} \mathrm{cm}^{-2}$ of the oxide film whose initial thickness was $27.5 \mathrm{~nm}$, where the potential, the amount of titanium ion dissolved from the oxide film, and the film thickness measured by ellipsometry are plotted as a function of galvanostatic reduction charge. In Fig. 2, it is seen that the dissolution of titanium ion and the reduction of film thickness begin to occur after an induction period has elapsed in which the potential is arrested at about $0.05 \mathrm{~V}$. When the potential reaches $-0.9 \mathrm{~V}$, the current efficiency for the oxide film reduction decreases and the complete removal of the oxide film thus becomes very difficult, probably because the applied cathodic current is consumed mostly by the hydrogen evolution reaction on the remaining oxide film and partially by the titanium hydride formation reaction.

Cathodic reduction in neutral solution.-During the reduction of the oxide film in neutral solution, an optical change in the film was observed without causing any dissolution of titanium ion from the oxide film. Figure 3 shows the ellipsometric parameters, $\Psi$ and $\Delta$, and the cathodic current observed during the potentiostatic reduction of the oxide film that has been formed at $7.65 \mathrm{~V}$ in phosphate buffer solution. In Fig. 3 , the recovery of $\Psi$ and $\Delta$ under the open-circuit condition after the cathodic current has been turned off is also shown. The cathodic current drops to $520 \mu \mathrm{A} \mathrm{cm}{ }^{-2}$ after an initial spike and then gradually increases, reaching a steady value of 650 $\mu \mathrm{A} \mathrm{cm}^{-2}$ in $0.5 \mathrm{~h}$. The ellipsometric parameters significantly change immediately after the potentiostatic reduction begins and they recover to the original values in $1.2 \times 10^{3} \mathrm{~s}$ under the open-circuit condition that follows. The similar behavior during the reduction was reported by Dyer et al. (3), who assumed the reversible hydrogen absorption into and desorption from the oxide film (3)

$$
\mathrm{H}^{+}{ }_{\text {ay }}+e \rightarrow \mathrm{H}_{\mathrm{ox}}
$$

Such changes of $\Psi$ and $\Delta$ as seen in Fig. 3 were measured at various cathodic potentials. Figure 4 shows the loci of $\Psi$ and $\Delta$ obtained after $0.5 \mathrm{~h}$ potentiostatic reduction at various potentials. The loci observed during cathodic reduction may reflect a change in the film composition caused by hydrogen absorbed in the film, as will be dis-

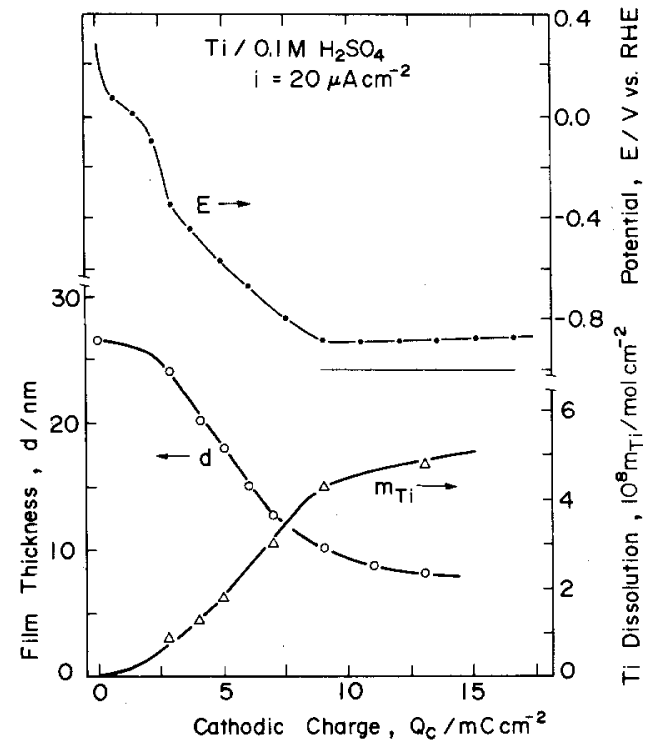

Fig. 2. Potential decay, film thickness, and amount of titanium ion dissolved from the oxide film during galvanostatic reduction at $i=-20$ $\mu \mathrm{A} \mathrm{cm}-2$ in $0.1 \mathrm{~mol} \mathrm{dm}^{-3}$ sulfuric acid solution. The original oxide film was anodically formed by potentiostatic th oxidation at $E=7.65 \mathrm{~V}$ (vs. $\mathrm{RHE}$ ) in phosphate buffer solution at $\mathrm{pH} 6.9$.

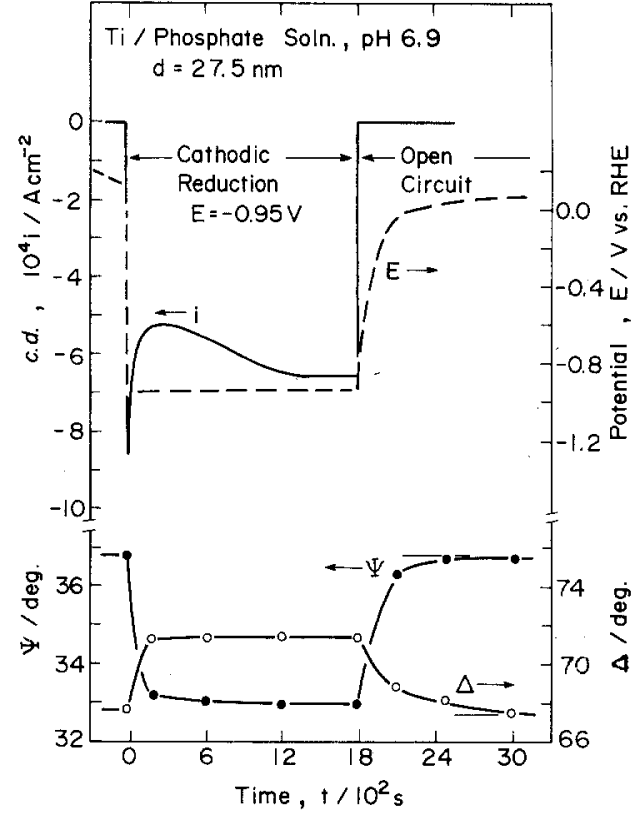

Fig. 3. Change in cathodic current and ellipsometric parameters $\Psi$ and $\Delta$ during potentiostatic reduction at $E=-0.95 \mathrm{~V}$ followed by open-circuit decay in phosphate buffer solution at $p \mathrm{H} \mathrm{6.9}$. The original film was anodically formed by potentiostatic $1 \mathrm{~h}$ oxidation at $E=$ $5.65 \mathrm{~V}$.

cussed later. If an anodic oxidation is applied following the cathodic reduction, the absorbed hydrogen in the oxide film can be released in accord with the reverse reaction of Eq. [1]. Figure 5 shows the change in anodic current with time during the reoxidation following the cathodic reduction. As seen in Fig. 5 , the current in an initial period of $100 \mathrm{~ms}$ strongly depends on the film thickness, increasing with increase of the film thickness. The anodic current continues to flow during the prolonged reoxidation and eventually reaches a small value almost independent of the film thickness. Figure 6 shows the dependence of current decay on the reoxidation potential at a constant film thickness of $d=27.5 \mathrm{~nm}$, where the initial decay of current for $160 \mathrm{~ms}$ is plotted in the form of $\log i$ vs. $t$ (Fig. 6A) and a prolonged decay in $\log i$ vs. $\log t$ (Fig. 6B). The initial decay may follow a linear relationship between $\log i$ and $t$. As the reoxidation is made at the higher potential, the very initial current is the larger,

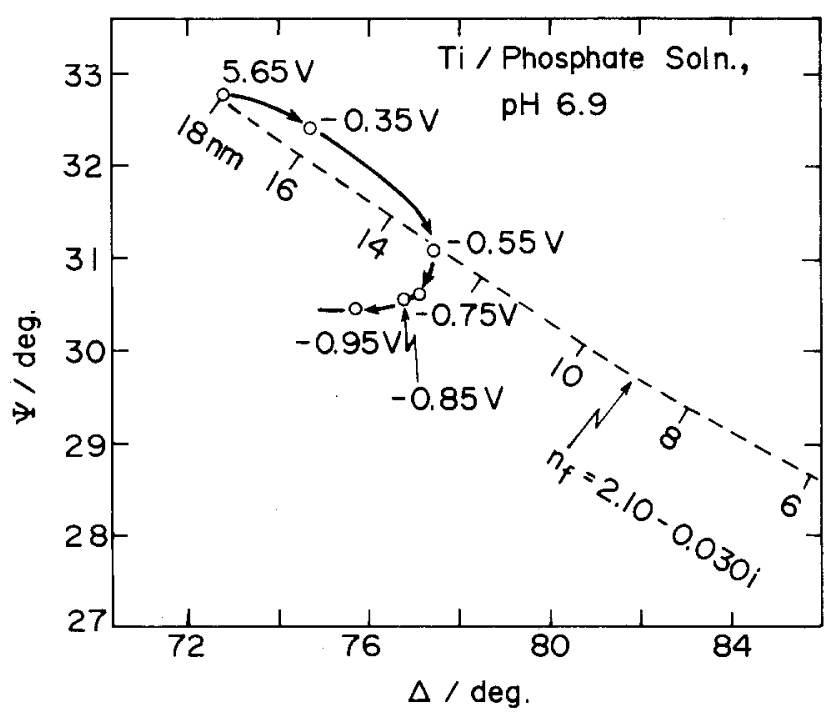

Fig. 4. Change in $\Psi$ and $\Delta$ as a function of cathodic potential. The values of $\Psi$ and $\Delta$ were obtained after $0.5 \mathrm{~h}$ reduction at various potentials in phosphate buffer solution at $p \mathrm{H} \mathrm{6.9}$. The original oxide film is the same as that in Fig. 1. 


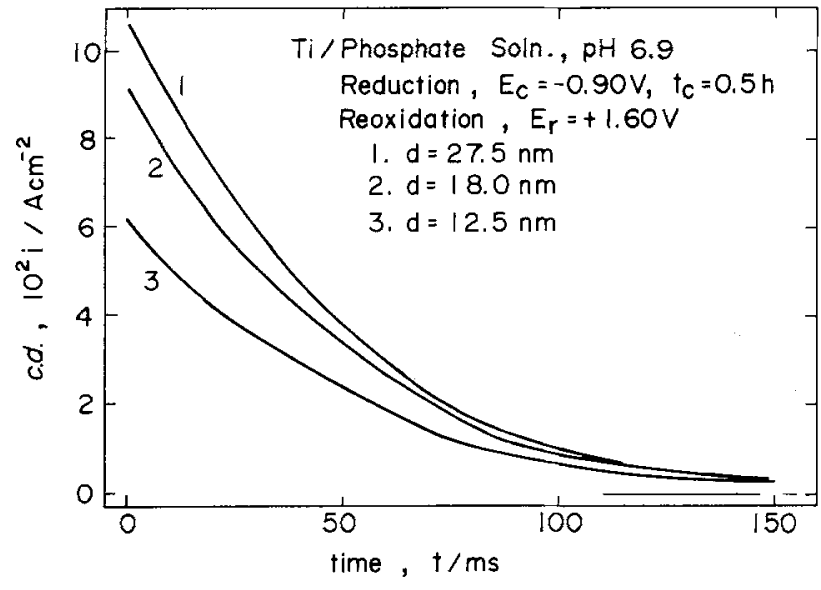

Fig. 5. Current decay during reoxidation at $1.60 \mathrm{~V}$ following cothodic reduction at $-0.90 \mathrm{~V}$ for $0.5 \mathrm{~h}$ of the anodic oxide films with various film thicknesses in phosphate buffer solution at $\mathrm{pH} 6.9$. The current was measured by a digital recorder.

decaying at the greater rate. The amount of charge estimated in the initial $1 \mathrm{~s}$ period is found to be about 6.3 $( \pm 0.6) \mathrm{mC} \mathrm{cm}^{-2}$ independent of the reoxidation potential. During prolonged reoxidation, the current decays, holding a linear relationship between $\log i$ and $\log t$ that is independent of the reoxidation potential. For the oxide film of $18.0 \mathrm{~nm}$ thickness, the similar relationship was observed between the current decay and the reoxidation potential, and the amount of charge in the initial 1s period following cathodic reduction at $-0.90 \mathrm{~V}$ for $0.5 \mathrm{~h}$ was found to be $5.7( \pm 0.4) \mathrm{mC} \mathrm{cm}^{-2}$ irrespective of the reoxidation potential.

Measurements were also made on the amount of charge during reoxidation for the films subjected to cathodic reduction at various cathodic potentials. The dependence of the amount of charge on the cathodic reduction potential will be discussed.

\section{Discussion}

Reductive dissolution in acidic solution.-During the reduction in acidic solution the anodic oxide film on titanium easily dissolves, and the amount of titanium ion dissolved into the solution depends on the applied cathodic charge, as shown in Fig. 1 and 2. Under the open- circuit condition, however, the anodic oxide film was gradually thinned at a rate of $1 \mathrm{~nm} / \mathrm{h}$. The results indicate that the anodic oxide film mainly composed of Ti(IV) oxide reductively dissolves to form $\mathrm{Ti}$ (III) ions in acidic solution

$$
\mathrm{TiO}_{2}+4 \mathrm{H}^{+}+e \rightarrow \mathrm{Ti}^{3+}{ }_{\mathrm{aq}}+\mathrm{H}_{2} \mathrm{O}
$$

In an induction period observed in the initial stage of reduction, no titanium ion is found in the solution, as shown in Fig. 2. Such an induction period during galvanostatic cathodic reduction has also been reported for the passivation film on iron (9). In the induction period, the cathodic charge is not consumed for the reductive dissolution of Ti(IV) $)_{0 \mathrm{x}}+e \rightarrow \mathrm{Ti}(\mathrm{III})_{\mathrm{a}, 1}$, but for some other cathodic charge transfer processes involving electron donor levels in the space charge layer in the semiconductive n-type oxide film.

Composition change due to hydrogen absorption.-In neutral solution, the cathodic reduction causes a change of the optical property of the oxide film, which obviously differs from the case of acidic solution where the cathodic reduction gives rise to the thinning of the oxide film without any change in its optical property. During the reduction, the absorption of hydrogen in the oxide film takes places in addition to the hydrogen evolution. The hydrogen absorption-desorption process during the reduction and the following reoxidation proceed reversibly, as shown in Fig. 3-6. The amount of hydrogen absorbed in the oxide film can be estimated from the measurement of the anodic charge for hydrogen desorption, which is equivalent to the charge passed during the reoxidation following the reduction. Figure 7 shows the results, where the reoxidation charge after the reduction at a constant potential of $-0.90 \mathrm{~V}$ ( $v \mathrm{~s}$. RHE) for $0.5 \mathrm{~h}$ is plotted as a function of the original film thickness. The reoxidation charge in the initial $1 \mathrm{~s}$ period is seen to increase linearly with the film thickness. This initial reoxidation charge, which is independent of the reoxidation potential, is assumed to be the amount of the hydrogen absorbed in the film. If the reoxidation progresses further, an extra charge of about $1 \mathrm{mC} \mathrm{cm}^{-2}$ passes in a time period of $10^{3} \mathrm{~s}$. This extra charge may be attributed to the desorption of hydrogen from the titanium substrate through the oxide film after the hydrogen in the film has been desorbed. As is shown in Fig. 6, the current decay curve in the initial $100 \mathrm{~ms}$ reoxidation obeys a linear relationship between $\log i$ and $t$, the slope of which depends on the reoxidation potential. The current decay curve in
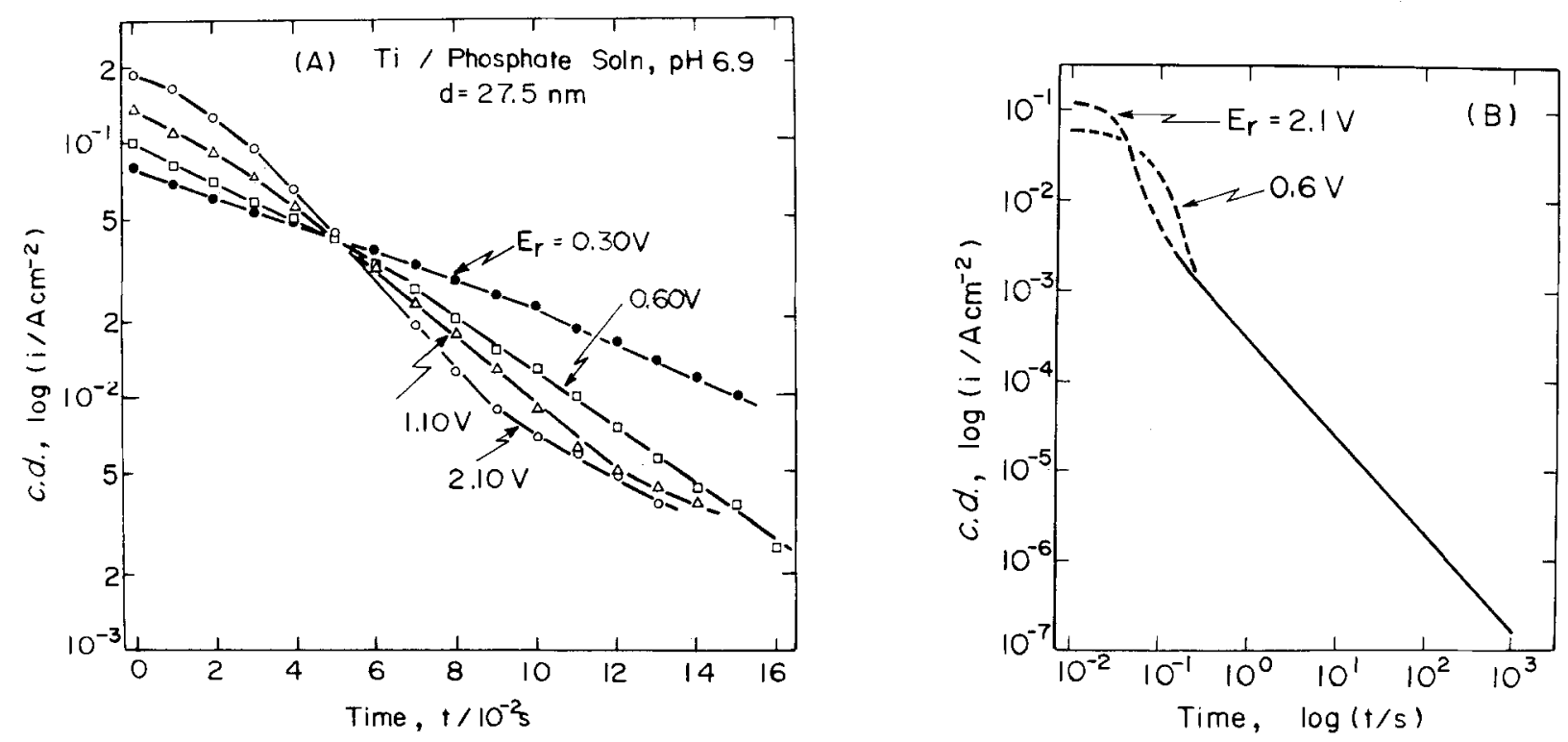

Fig. 6. Current-time relationship during reoxidation at various anodic potentials following cathodic reduction at $-0.90 \mathrm{~V}$ for $0.5 \mathrm{~h}$ in neutral phosphate buffer solution. The original thickness of the oxide film formed at $7.65 \mathrm{~V}$ for $1 \mathrm{~h} \mathrm{is} 27.5 \mathrm{~nm}$. (A) Relationship of log i vs. $t$ during the initial stage of reoxidation at potential of $E=0.30,0.60,1.10$, and $2.10 \mathrm{~V}$. (B) Relationship of $\log i$ vs. $\log t$ during prolonged reoxidation at $E=0.60$ and $2.10 \mathrm{~V}$. 


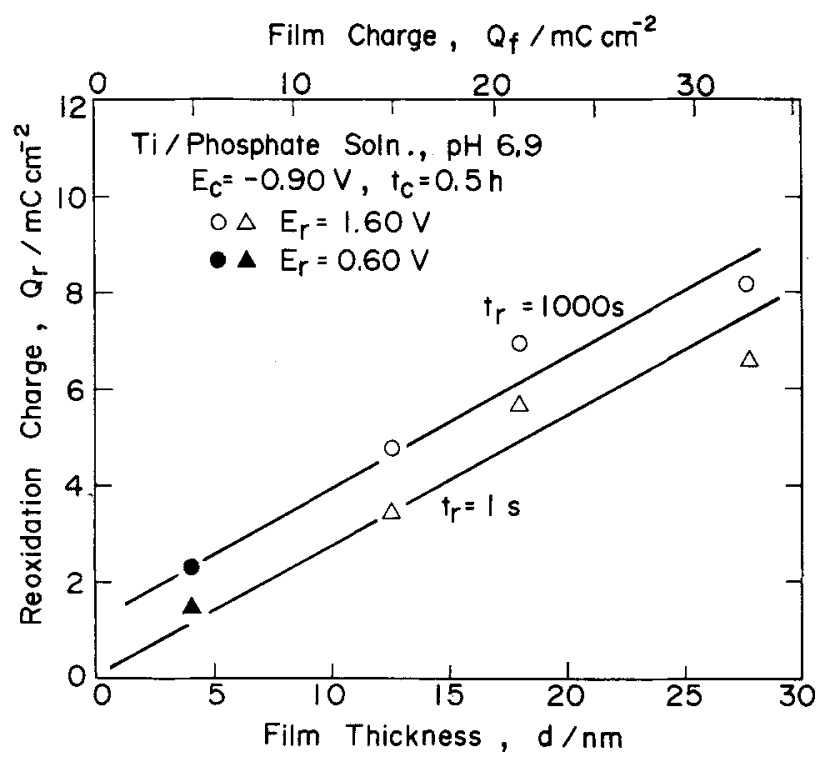

Fig. 7. Anodic charge passed during reoxidation at $E=1.60$ or $0.60 \mathrm{~V}$ following cathodic reduction at $E=-0.90 \mathrm{~V}$ in neutral phosphate buffer solution as a function of the film thickness. The upper scale in the figure indicates the anodic charge accumulated during anodic formation of the film.

the prolonged reoxidation after the initial $1 \mathrm{~s}$ period, however, obeys a linear relationship between $\log i$ and $\log t$, the slope of which is independent of the reoxidation potential. The reoxidation reaction during the hydrogen desorption may thus include two processes: one is the desorption of hydrogen from the film, which proceeds at a relatively high rate, and the other is the desorption of hydrogen from the substrate titanium through the film, which takes place at a relatively low rate. The desorption mechanism, which may be discussed from the current-time relationship (10), will be presented in a separate paper. If the reoxidation charge, $Q_{r}$, in the initial is period ${ }^{1}$ is equivalent to the amount of hydrogen absorbed in the film, and if the anodic charge accumulated during the film formation is obtained from the film thickness-to-anodic charge ratio of $\left(d / Q_{f}\right)=0.85$ $\mathrm{nm} \mathrm{cm} / \mathrm{mC}(1)$, one can calculate the ratio of the number of hydrogen atoms absorbed to the number of titanium(IV) ions present in the oxide film. In Fig. 7, the upper scale indicates the anodic charge $Q_{\mathrm{f}}$ calculated from the film thickness-to-anodic charge ratio: The slope of the straight line in Fig. 7 corresponds to a value of $\left(Q_{\mathrm{r}} / Q_{\mathrm{f}}\right)$ $=0.25$, which indicates that one atom of hydrogen is absorbed for one Ti(IV) ion in the film according to the reaction of $\mathrm{TiO}_{2}+\mathrm{H}^{+}+e \rightarrow$ TiOOH at the potential of $-0.90 \mathrm{~V}$, independent of the film thickness. From the reoxidation charge, $Q_{r}$, for the oxide film reduced at various cathodic potentials, the composition change of the oxide film during the reduction can be evaluated as a function of the cathodic potential, in which the following cathodic reaction is assumed

$$
\mathrm{TiO}_{2}+x \mathrm{H}^{+}{ }_{\text {aq }}+x e \rightarrow \mathrm{TiO}_{2-x}(\mathrm{OH})_{s}
$$

The results are given in Fig. 8 for the oxide films $18.0 \mathrm{~nm}$ and $27.5 \mathrm{~nm}$ thick. In Fig. 8, the complex refractive index of the film is also plotted. For calculation of the complex refractive index of the film from the ellipsometric data shown in Fig. 4, the constant film thickness has been as sumed, because the hydrogen absorbed exerts no in fluence on the film thickness. In Fig. 8, it is seen that the composition begins to change at $-0.25 \mathrm{~V}$ and reaches TiOOH at $E=-0.90 \mathrm{~V}$. Simultaneously, the complex re-

'It is difficult to determine the exact transient point in time from the initial reduction stage to the prolonged stage. Therefore, we assume that the amount of charge passed during the initial is period corresponds to the amount of desorbed hydrogen from the film.

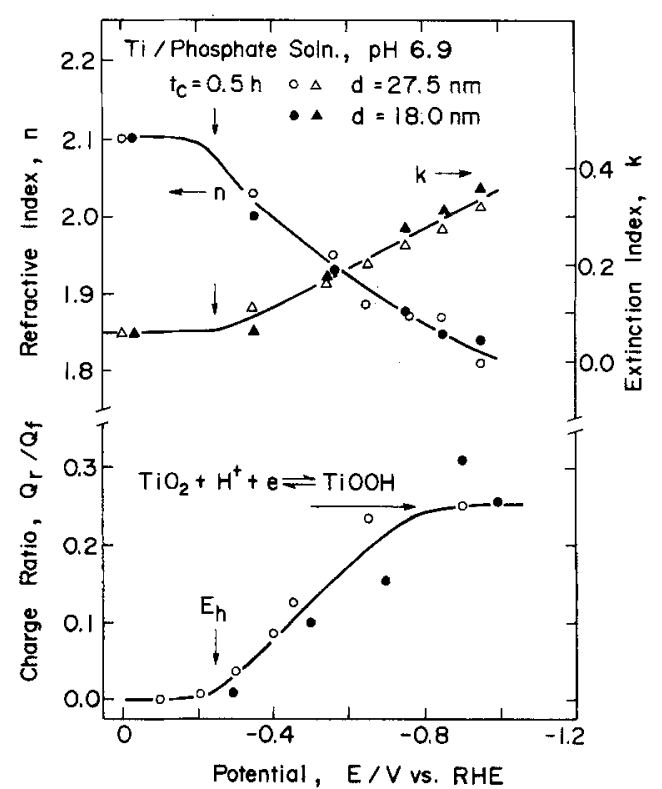

Fig. 8. Change in composition and refractive index of the anodic oxide film on titanium during potentiostatic reduction for $0.5 \mathrm{~h}$ in neutral phosphate buffer solution as a function of cathodic potential.

fractive index changes from the value of anodic oxide film of $n_{\mathrm{F}}=2.3-0.03 \mathrm{i}$ to the value of $n_{\mathrm{f}}=1.85-0.35 \mathrm{i}$.

The decrease of the refractive index (the real part of the complex refractive index) and the increase of the extinction index (the imaginary part of the index) due to the reduction were also seen in an early observation made by Dyer et al. (3). The change of the indexes due to hydrogen absorption may be explained in the following way. The introduction of hydrogen into the film increases the concentration of $\mathrm{OH}$ bonding, resulting in a decrease of polarizability of $\mathrm{O}^{2-}$ ions and thus a decrease of the refractive index. The hydrogen in the film can act also as an electron donor

$$
\mathrm{H} \rightarrow \mathrm{H}^{+}+e
$$

and produces a new absorption band in the bandgap re gion of the anodic oxide film. The hydrogen absorption, therefore, causes an increase of the light absorption or of the extinction index.

\section{Conclusion}

1. In acidic solution, the cathodic reduction of the anodic oxide film on titanium causes the reductive dissolution of the film in accordance with the reaction of $\operatorname{Ti}(\mathrm{IV})_{\mathrm{ox}} \rightarrow \operatorname{Ti}(\mathrm{III})_{\mathrm{aq}}+e$.

2. In neutral solution, the cathodic reduction of the film does not cause the film dissolution but gives rise to a change of film composition due to the hydrogen absorption, which is represented by the reaction of $\mathrm{TiO}_{2}+x \mathrm{H}^{+}$ $+x e \rightarrow \mathrm{TiO}_{2-r}(\mathrm{OH})_{x}$. The amount of hydrogen absorbed for a unit volume of the film is a function of the cathodic potential applied. This composition change is accompanied by a marked change of the optical property of the film.

Manuscript submitted April 23, 1986; revised manuscript received March 23, 1987

Hokkaido University assisted in meeting the publication costs of this article.

\section{REFERENCES}

1. T. Ohtsuka, M. Masuda, and N. Sato, This Journal, 132, 787 (1985).

2. T. Fukuzuka, K. Shimogri, H. Satoh, F. Kamikubo, and H. Hirose, Boshoku Gijutsu, 26, 490 (1979).

3. C. K. Dyer and J. S. L. Leach, This Journal, 125, 23 (1978). 
4. C. K. Dyer and J. S. L. Leach, Electrochim. Acta, 23 1387 (1978)

5. M. F. Weber, L. Schumacher, and M. J. Dignam, This Journal, 129, 2022 (1982).

6. D. S. Ginley and M. L. Knotek, This Journal, 126, 2163 (1979).
7. T. Ohzuku and T. Hirai, Electrochim. Acta, 27, 1263 (1982).

8. L. A. Harris, This Journal, 127, 2657 (1980).

9. M. Nagayama and M. Cohen, ibid. 109, 781 (1962).

10. J. Vondrak, Electrochim. Acta, 29, 1191 (1984); ibid., 32, 163 (1987)

\title{
A Photocurrent Spectroscopic Investigation of Passive Films on Ferritic Stainless Steels
}

\author{
C. Sunseri, S. Piazza, A. Di Paola, and F. Di Quarto \\ Istituto di Ingegneria Chimica, Università di Palermo, Viale delle Scienze, 90128 Palermo, Italia
}

\begin{abstract}
An investigation of the surface films on three different ferritic stainless steels was carried out by means of photocurrent spectroscopy. All the specimens showed detectable photoeffects only after long anodic polarization times in chloride ion-containing solution. The passive films showed n-type behavior and bandgap values dependent upon the applied potential. A difference was observed in the bandgap values of films grown on different alloys. The photoelectrochemical behavior of the films is compared to that of passive layers on pure iron and chromium. Due to the lack of photoelectrochemical data on passive films on $\mathrm{Cr}$ a preliminary investigation on this metal has been also carried out. The experimental results are interpreted in terms of formation of different iron-chromium oxide solid solutions.
\end{abstract}

The development of new processing techniques has recently enhanced the commercial importance of the ferritic stainless steels. In fact the decrease of $\mathrm{C}$ and $\mathrm{N}$ interstitials, together with the stabilization due to $\mathrm{Nb}$ and $\mathrm{Ti}$ has made these materials very attractive because of their high corrosion resistance in many environments and their lower cost with respect to the austenitic steels. For these reasons ferritic stainless steels have been suggested as replacements for the austenitic alloys in some applications such as condensers and heat-exchangers for sea water service. Such a substitution is partially hindered by the lack of information concerning the behavior of the new ferritic stainless steels in industrial service, while a lot of data is available for the austenitic ones. Consequently, in the last few years many studies have been undertaken to reach a better knowledge of the mechanical and corrosion properties of extra low interstitial (ELI) ferritic stainless steels (1-10).

On the other hand, it is known that the nature of passive films on metals and alloys is the ultimate factor which controls their corrosion behavior. For these reasons in recent years much work has been devoted to the study of the chemico-physical properties of passive layers on metals and alloys $(11,12)$.

Many powerful analytical tools (Auger, XPS, ISS, SIMS, etc.) have been employed in order to gain a better knowledge of the nature of corrosion product layers. The main drawback to the use of such techniques in the corrosion field is that they are ex situ methods requiring high vacuum systems. The information obtained is affected by the change of environment which occurs on going from the electrochemical cell to the vacuum chamber.

On the other hand, several in situ methods have been suggested as analytical tools for studying such films. Many in situ methods are based on the study of the interaction between light and matter. In the case of corrosion product layers having semiconducting or insulating behavior it has been shown by several authors (13-18) that useful information on the nature and growth of the passive films can be obtained by means of photocurrent spectroscopy (PCS). The use of such a technique in the field of corrosion requires the extension of concepts and theories valid for crystalline materials but which can be misleading in the case of amorphous or strongly disordered passive films. Further complications are related to the fact that in many practical applications the passive films grown on alloys are not homogeneous both at large as well as at microscopic scale so that the information obtained is often qualitative.

The aim of this work is to test the usefulness of photocurrent spectroscopy in the study of passive films on complex systems like stainless steels which are perhaps the most widely used alloys in industrial applications.

In an attempt to relate the corrosion behavior to the nature of passive films Bianchi et al. (19) showed that the susceptibility to pitting nucleation can be related to the semiconducting properties of oxide films formed on austenitic stainless steels.

In a previous work (20) we have also shown that the different photoelectrochemical behavior exhibited by ferritic and superaustenitic stainless steels in a large range of electrode potentials could be traced to the different nature of passive films on different alloys.

In this work we present new results on the nature of passive films grown on ferritic stainless steels having slightly different compositions with respect to that previously investigated but which have been submitted to a different electrochemical treatment. The experimental results will also be discussed by taking into account the corrosion behavior of such alloys.

\section{Experimental}

The ELI ferritic stainless steels were prepared by C. S. M. (Centro Sperimentale Metallurgico-Roma). The chemical composition of the samples is shown in Table I.

The samples were cut in rectangular sheets and sealed with epoxy resin leaving only a planar surface in contact with the solution. Electrical back contacts were ensured by mechanical pressure. The electrodes were polished with fine grained abrasive paper, rinsed with distilled water, and ultrasonically cleaned. After drying the electrodes were exposed to air for about ih before introducing them into the electrolytic cell. The absence of any photoeffect for a very long time (see below) after immersion of the electrodes in solution ensures that the observed photoelectrochemical behavior has to be related to the passive films formed in solution. The films were grown at a maximum potential of $0.6 \mathrm{~V}$ (SCE) in order to avoid chromium depletion which is reported to occur at higher potentials (21).

Photoelectrochemical experiments were performed by using a $150 \mathrm{~W}$ xenon lamp coupled to a grating monochromator (Bausch \& Lomb). The sign of the photoeffect was detected recording the change in the total current caused by an intermittent illumination. Because of the 